\title{
Enantiomeric Recognition in Host-Guest Complexation Using Chiral Bis-pyridino- 18-crown-6 Ethers, by Electrospray Ionization Mass Spectrometry (ESI-MS) Enantiomer-Labelled (EL) Guest Method
}

\author{
Jae-kon Kim, Jung ju Seo, Eui Soon Yim, ${ }^{\ddagger}$ Youngeup Jin, Suhee Song, and Hongsuk Suh ${ }^{\star}$ \\ Deparment of Chemistrv and Chemistr. Institute for Functional Materials, Pusan National Cniversin, Pusan 609-735, Korea \\ E-mail: hssuhiàpusan ac.kr \\ ${ }^{\dagger}$ Korea Basic Science Institute, Seoul 136-713, Korea \\ ${ }^{\ddagger}$ Department of Chemistry, Chungnam National Chiversitw. Daejeon $305-764$. Korea \\ Received Wovember 22. 2007
}

Key Words : Bis-pyridino-18-crown-6 ether, Enantiomeric recognition. Electrospray ionization mass spectrometry (ESI-MS), Enantiomer-labeled (EL) guest method

Enantiomeric recognition of chiral amino acids by ș̣nthetic and natural host compounds is one of the most challenging subjects in modern host-guest chemistry. ${ }^{3}$ Hostguest chiral recognition plays an important role in biological process resolution of enantiomers. and asymmetric cataly sis reactions. 2 A number of sy'nthetic model compounds have been designed and synthesized as chiral host molecules that help chemists understand the basis of the mechanism of host-guest complexations and their chiral recognitions. Hence. the successful design. synthesis and application of chiral macrocyclic ligands with the selective recognition of the guests have attracted much attention for the investigations of catalysis. ${ }^{3}$ separations. ${ }^{4}$ and enzy'me mimics. ${ }^{5}$ To determine the chiral recognition of these hosts. various methods of extraction/NMR extraction/polarimetry. NMR titration. variable temperature NMR nuclear overhauser effects (NOE). UV-ris titration. HPLC. capillary electrophoresis transport. and membrane electrode have been used. ${ }^{6}$ A high degree of chiral recognition was observed in these studies.

In the host-guest complex systems, chiral recognition has been detected by Sawada and his co-workers using fast atom bombardment mass spectrometry (FAB-MS) ${ }^{7}$ and electrospray ionization mass spectrometry (ESI-MS). ${ }^{8}$ In this reports. ESI-MS enantiomer-labeled (EL) guest method is utilized. This method requires the isotopic labeling of guest $\left(\mathrm{G}^{-}\right)$enantiomers and detects the complexation of a target host $(\mathrm{H})$ compound with a 1:1 mixture of the unlabeled $\left(\mathrm{G}_{R}{ }^{+}\right)$and labeled enantiomer guests $\left(\mathrm{G}_{S-6+s_{1}}\right)$. The peak intensity ratio. $I\left[\left(\mathrm{H}+\mathrm{G}_{R}\right)^{-}\right] / I\left[\left(\mathrm{H}+\mathrm{G}_{\mathrm{S}} \cdot(\mathrm{m})\right.\right.$ ). of two diasteromeric host-guest complex. which appeared simultaneously with $n$ mass-unit difference in one ESI mass spectrum. was abbreviated as 'IRIS" for shot and adopted here as a critical measure for detecting chiral recognition ability. This method is a direct and operationally simple method and it is a major feature of the stream needed for rapid screening of enantiomeric recognition.

We studied the synthesis of chiral bis-pyridino-18-crown6 ethers ${ }^{9}$ and the chiral recognition of $\alpha$-amino acids and chiral amines by FAB-MS EL guest method. ${ }^{9 a}$ UV-ris titra- tion, ${ }^{9 \mathrm{~h}}$ and ${ }^{\mathrm{l} H} \mathrm{H}-\mathrm{NMR}$ titration. ${ }^{9 \mathrm{c}}$ Our interest has been focused on the enantiomeric recognition of amino acids by utilizing synthetic chiral bis-pyridino-18-crown-6 ether. We report herein the synthesis of chiral bis-pyridino-18-crown-6. $(R R R R)-5$ and 6 with tetraethyl tetracarboxylate and tetramethyl tetracarboxamide groups as chiral barriers. and their enantiomeric recognition of several amino acid methyl ester hydrochlorides (7-16) by ESI-MS EL guest method The IRIS values for the enantiomeric recognition of amino acid methyl ester hydrochlorides (7-16) using chiral bispyridino-18-crown-6 ether. $(R, R, R R)-5$ and 6 . were detected by ESI-MS EL guest method.

\section{Experimental Section}

General. 'H-NMR and ${ }^{13} \mathrm{C}-\mathrm{NMR}$ spectra were recorded on a Varian Unity Plus $5(500 \mathrm{MHz})$. High-resolution mass spectra (HRMS) were recorded on a JEOL JMS-700 mass spectrometer under fast atom bombardment (FAB) conditions with nitrobenzyl alcohol (NBA) as the matrix. in the Korea Basic Science Institute (Seoul), Korea. Column chromatography was performed using E. Merck silica gel (60. particle size 0.040-0.063 mm) and alumina 60 (Merck. 70-230 mesh). Analytical thin layer cluromatographỵ (TLC) was performed using pre-coated TLC plates with silica Gel $60 \mathrm{~F}_{3-4}(\mathrm{E}$. Merck no. 5715-7) and alumina 60 (Merck. 70230 mesh). All reactions were carried out under argon atmosphere with dry solvent. unless otherwise noted. Dimethyl formamide (DMF) were distilled from sodiunv benzophenone immediately prior to use and acetone was dried from calcium chloride.

All chemicals were reagent grade unless otherwise specified. Commercial samples (Sigma-Aldrich) of $(D)$-methyl alaninate hydrochloride. (D)-methyl methionate hydrochloride were used without purification. All other amino acid methyl ester hydrochlorides were synthesized and purified according to the standard ${ }^{7}$ methods using commercially available (Sigma-Aldrich) $(D)$-amino acids and $(L)$-amino acids. In case of $(S)$-amino acids the amino acids were treated $\mathrm{CD}_{2} \mathrm{OD}$ and anhydrous hydrochloride gas to generate 
the isotopic labelled $\left(S\right.$ - $\left.d_{3}\right)$-amino acid methyl ester hydrochlorides. The 2.6-bis(iodomethyl)pyridine (3). ${ }^{\text {gil }}$ 2.6-bis(benzenesulfonylmethyl)pyridine (4) was prepared using the reported methods. ${ }^{11}$

Synthesis of Tetraethyl $(+R, 5 R, 15 R, 16 R)-3,6,14,17$-tetraoxa-23,24-diazatricyclo[17.3.1.1 ${ }^{8,12}$ |tetracosa-1(23),8(24), 9,11,19,21-hexaene-4,5,15,16-tetracarboxylate (5). To a stirred solution of diethyl- $L$-tartrate $(1)(7 \mathrm{~g}, 33.95 \mathrm{mmol})$ in dry DMF $(700 \mathrm{ml})$ at room temperature under argon was added thallous ethoxide ( $16.9 \mathrm{~g} .67 .9 \mathrm{mmol}$ ) over a period of $15 \mathrm{~min}$ and the resulting white suspension was stirred for 30 min. A solution of 2,6-bis(iodonethyl)pyridine (3) (16 g. $52.28 \mathrm{mmol})$ in dry DMF $(50 \mathrm{~mL})$ was added dropwise and the mixture stirred for a further $1 \mathrm{~h}$. Then temperature was raised to $70{ }^{\circ} \mathrm{C}$ in about $\mathrm{l} \mathrm{h}$ and maintained at that temperature for $12 \mathrm{~h}$. The orange thallous iodide was removed by filteration through a pad of sand (3 $\mathrm{cm}$ height) over alumina ( $5 \mathrm{~cm}$ height) on Buchnner funnel, and concentrated under reduced pressure. The residue was purified by chromatography on alumina 60 (Merck, $70-230$ mesh. $R_{f}$ $\left.0.31,100 \% \mathrm{CH}_{2} \mathrm{Cl}_{2} \rightarrow 3 \% \mathrm{MeOH}-\mathrm{CH}_{2} \mathrm{Cl}_{2}\right)$ to give $(R, R R R)-$ $5(4.40 \mathrm{~g}, 21 \%)$ as a pale yellow oil.

$[\alpha]_{\mathrm{D}}^{2+4}=+78.5^{\circ}\left(\mathrm{c}=0.1 . \mathrm{CHCl}_{3}\right) ;{ }^{1} \mathrm{H}-\mathrm{NMR}(500 \mathrm{MHz}$. $\left.\mathrm{CDCl}_{3}\right) \delta 1.32$ (t. $\left.12 \mathrm{H}, J=6.8 \mathrm{~Hz}\right) .4 .16-4.28(\mathrm{~m}, 8 \mathrm{H}), 4.4$ $(\mathrm{s} .4 \mathrm{H}) .4 .7(\mathrm{~s}, 8 \mathrm{H}) .7 .27$ (d. 4 H. $J=6.6 \mathrm{~Hz}) .7 .60$ (t. $2 \mathrm{H}, J$ $=7.6 \mathrm{~Hz} ;{ }^{13} \mathrm{C} \cdot \mathrm{NMR}\left(125 \mathrm{MHz}, \mathrm{CDCl}_{3}\right) \delta 14,0,61.4,73.5$. 79.2, 120.8, 137.1. 156.8. 169.0. HRMS (FAB, NBA) calcd 619.2503 for $\mathrm{C}_{31 !} \mathrm{H}_{3 \times} \mathrm{N}_{2} \mathrm{O}_{12}\left(\mathrm{M}+\mathrm{H}^{-}\right.$, found 619.2499 .

\section{Results and Discussion}

Since the symunetry of macrocycle is crucial for chiral molecular recognition, we focused on synthesis of $C_{3}$ symumetirc chiral bis-pyridino-18-crown-6 ether $(R, R, R . R)-5$ and 6. The synthesis of $(R R, R R)-5$ and 6 is sunumarized in Scheme 1. The new chiral bis-pyridino-18-crown-6 ether $(R R, R, R)-5$ and 6, substituted with tetraethyl tetracarboxylate and tetramethyl tetracarboxamide groups as chiral barriers. were selected as the host compound. The synthesis of $(R, R R R)-5$ and 6 is based on a modified Willamson synthesis of ethers derived that described by Seebach $e t a l^{12}$ Chiral bis-pyridino-18-crown-6 ether $(R, R, R, R)-5$ and $6^{1 \mathrm{i}}$ were prepared from diethyl- $L$-tartrate (1) or $N, N, N^{\prime} N^{\prime}$ -

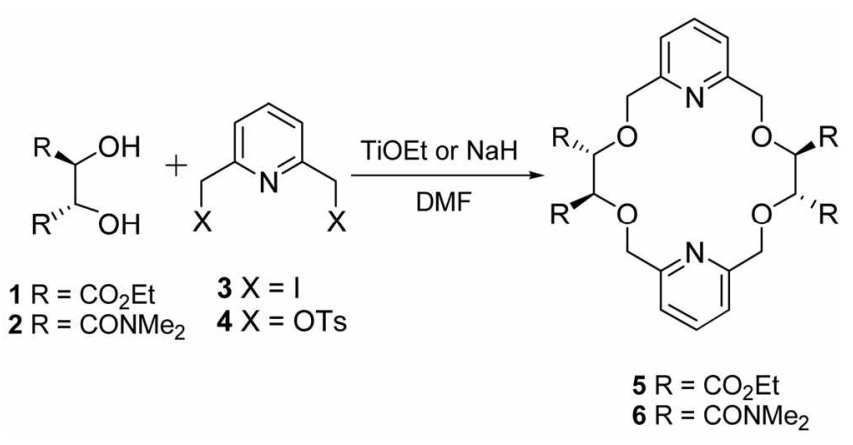

Scheme 1. Synthetic Routes for Chiral Bis-pyridino-18-crown-6 Ethers.

tetramethyl tartaramide (2) with 2,6-bis(iodomethyl)pyridine (3) in the presence two equivalent of thallous ethoxide as the base under high dilution conditions. which yields are $19-21 \%$ respectively. However. when the sodium hydride as the base was used in the synthesis of $(R R, R R)-5$ and 6 . the yield dropped in $8-9 \%$. respectively. When the di- $p$ toluenesulfonate 4 was used, instead of the diiodide 3 , in the synthesis of $(R R R R)-5$ and 6. the yield also decreased from $21 \%$ to $7 \%$. and no appreciable alkylation with the corresponding dichloride occurred. The structures of $(R, R, R, R)-\mathbf{5}$ and 6 were identified by ${ }^{1} \mathrm{H}-\mathrm{NMR},{ }^{13} \mathrm{C} \cdot \mathrm{NMR}$, and FABMS.

The enantiomeric recognition of amino acid methyl hydrochlorides by chiral bis-pyridino-18-crown- 6 ether $(R R R R)$ 5 and 6 have been evaluated by ESI-MS EL guest method. We used $(R R R R)-5$ and 6 as the host and amino acid methyl ester $\left(\mathrm{CH}_{3}\right.$ and $\left.\mathrm{CD}_{3}\right)$ hydrochlorides (7-16) as the guest in Figure 1 . The IRIS values, $I\left[\left(\mathrm{H}+\mathrm{G}_{R}\right)^{-}\right] / I\left[\left(\mathrm{H}+\mathrm{G}_{\text {s- }}\right.\right.$ $\left.\left.\alpha_{i}\right)^{-}\right]$, were detected by the ESI-MS EL guest method using various amino acid methyl ester hydrochlorides were summarized in Table 1 . and the values are corrected by the natural abundance of the $(\mathrm{M}+3)$ isotope. IRIS values in Table $\mathrm{I}$ can be classified as following three types. (1) IRIS $>1.0$ means that a given chiral host binds more strongly the $(R)$ enantiomer of a given guests: $(R)$-enantiomer preference. The larger the $I_{k} / I_{\text {s-tat }}$ value from unity. the higher the degree of chiral recognition of the host. (2) In contrast. $I R I S<1.0$ means that a given chiral host binds more strongly the (.S)enantiomer of a given guests: (S)-enantiomer preference. (3) $I R I S=1.0 \pm 0.05$ means that a given chiral host compound<smiles>[R]OC(=O)C(C)[NH3+]</smiles><smiles>[R]OC(=O)C([NH3+])C(C)C</smiles>

$\mathrm{AlaOMe}^{+}(\mathbf{7})$

$$
\mathrm{ValOMe}^{+}(8)
$$<smiles>[R]OC(=O)C([NH3+])CCSC</smiles><smiles>[R]OC(=O)C([NH3+])c1ccccc1</smiles>

$\mathrm{PGlyOMe}^{+}(\mathbf{1 4})$<smiles>[R]OC(=O)C([NH3+])CCCC</smiles>

NorleuOMe ${ }^{+}(\mathbf{1 0})$<smiles>[R]OC(=O)C([NH3+])Cc1ccccc1</smiles>

$\mathrm{PheOMe}^{+}(15)$<smiles>[R]OC(=O)C([NH3+])CC(C)C</smiles>

LeuOMe ${ }^{+}$(11)

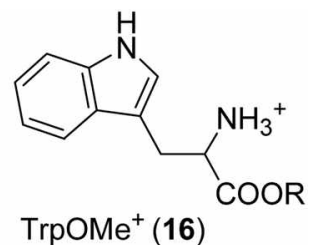

Figure 1. The chemical structure of amino acid methyl ester hydrochlorides $\left(\mathrm{CH}_{3}\right.$ and $\mathrm{CD}_{3}$ esters) (7-16) used in experiments. 


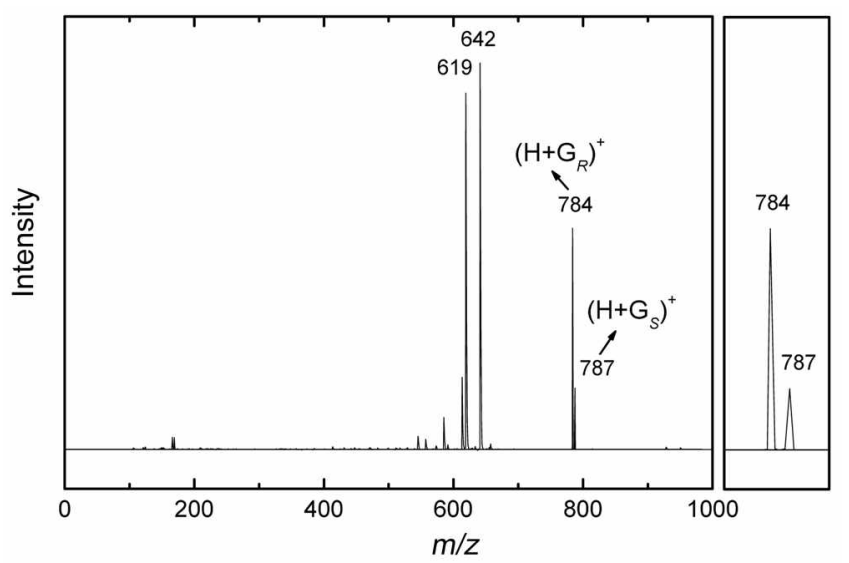

Figure 2. An expansion of the ESI mass spectrum for the complexation between $(R R R R)-5$ and $1: 1$ misture of $(R)-13$ and $(S)-13-d ;$

cannot differentiate the chirality of the given guests. ${ }^{8}$ The representative ESI-MS spectrum for the complexation between $(R, R, R, R)-5$ and $1: 1$ mixture of $(R)-13$ and $(S)-13-d_{3}$ was shown in Figure 2. As shown in the Table 1, the $(R, R, R R)-5$ exhibited $I R I S$ values range from 1.30 to 7.66 . and showed $(R)$-enantiomer preference toward all guests. $(R R, R, R)-5$ also showed the highest degree of $(R)$-enantiomer predominance toward guest 7 in this experiments. Especially. $(R, R, R, R)-5$ showed a characteristic pattern when $\mathrm{R}$ is only primary alkyl group (guests 7,9.10 and 13) in amino acid methyl ester lydrochlorides ( $\mathrm{RCH}(\mathrm{COOMe}) \mathrm{NH}_{3}{ }^{+}$). As $R$ alkyl chain length increases (guest $7 \rightarrow$ guest 13 ). IRIS values showed a slight decline $(7.66 \rightarrow 1.94)$. In the same way, $(R R R R)-6$ evaluated from 0.22 to 2.31 in $I R I S$ values for chiral recognition, and showed inconsistent preference as compared with $(R R R R)-\mathbf{5}$.

The origin of the enantiomeric recognition for the complex between host and guest has been estimated on the basis of complex ion structure. We performed molecular modeling and an optimization of these complexes by Spartan ' 02 software with semi-empirical force (PM3). ${ }^{i}$ The minimized conformations of the complexes between $(R R R R)-5,6$ and LeuOMe ${ }^{+}$(11) are shown in Figure 3. The complexes are basically possible to have tripod hydrogen bonding between the one nitrogen and two oxy'gen of the host and three hydrogen of the ammonium cation of the guest. ${ }^{9}$ As expected from PM3 calculation. tetraethyl tetracarboxylate group of $(R R R R)$ 5 is located almost upper side relative to the macrocyclic ring plane in Figure 3. Since $(R R R R)-5$ is expected to be
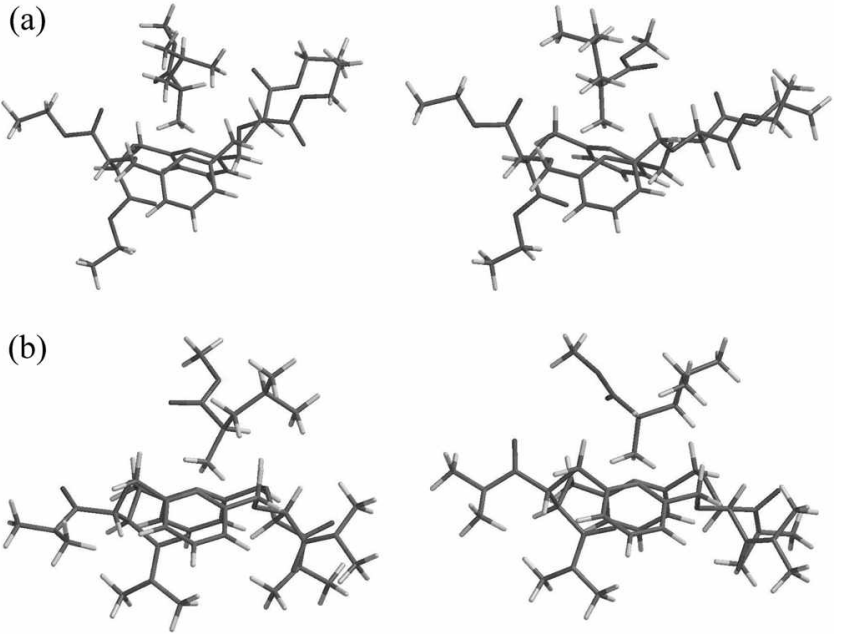

Figure 3. Structures of the diasteromeric host-guest complex ions between host $(R R R R)-5,6$ and guest LeloMe ${ }^{+}$(11) estimated from PM3 calculations (a side view): (a) host $(R R R R)-5$ plus guest $(R)-11$ (left) and host $(R R, R, R)-5$ plus $(S)-11$ (right), (b) host $(R R R R)-6$ plus guest $(R)-11$ (left) and host $(R R R R)-6$ plus $(S)-$ 11 (right).

conformationally less mobile than $(R, R, R R)-\mathbf{6}$, tetraetlyy tetracarboxylate group will effectively block the complexation space. and serve as an efficient chiral barrier for the complexation. It is indicated that the complexes with $(R . R R R)$-5 have more steric repulsion between the alkyl or aromatic group on the chiral carbon of the amino acid methyl ester hydrochloride and tetraethyl tetracarbosylate of the host as compared with tetramethyl tetracarboxamide of the $(R, R R R)-6$. Based on the molecular mechanics and experimental result, it is possible to say that $(R R, R R)-5$ forms more stable complex and shows enantiomeric recognition toward amino acid metlyl ester hydrochlorides as compared to the case of $(R R R R)-6$.

In conclusion, the synthesis and enantiomeric recognition of chiral bis-pyridino-18-crown-6 ether. $(R R R R)-\mathbf{5}$ and $\mathbf{6}$. with tetraethyl tetracarboxylate and tetramethyl tetracarboxamide groups as chiral barriers are reported. The IRIS values for the enantiomeric recognition of amino acid methyl ester hydorchlorides (7-16) using chiral bis-pyridino-18-crown-6 ether. $(R, R R R)-5$ and 6 . were detected by electrospray ionization (ESI) mass spectrometry (MS) with the enantionerlabeled (EL) guest method. $(R R R R)-\mathbf{5}$ forms more stable complexes and has a good enantiomeric recognition toward anino acid methyl ester hydrochlorides as compared to the case of $(R R \cdot R R)-6$.

Table 1. IRIS Values" using the ESI-MS Guest Method ${ }^{\circ}$

\begin{tabular}{|c|c|c|c|c|c|c|c|c|c|c|}
\hline \multirow{2}{*}{ Host } & \multicolumn{10}{|c|}{ Gulest ${ }^{\prime}$} \\
\hline & 7 & 8 & 9 & 10 & 11 & 12 & 13 & 14 & 15 & 16 \\
\hline 5 & 7.66 & 1.65 & 3.41 & 2.36 & 5.59 & 1.30 & 1.94 & 4.17 & 1.80 & 3.44 \\
\hline 6 & 0.22 & 0.82 & 2.27 & 0.52 & 1.06 & 2.31 & 0.81 & 0.52 & 1.68 & 2.23 \\
\hline
\end{tabular}

"The values are corrected by the natural abundance of the comesponding $(\mathrm{M}-3)$. For one measurement. the IRIS value was obtained from an average of those of three times (Nos. 10. 20. 30). ${ }^{b}$ Concentration condition: $\left[\mathrm{G}^{-}\right]=0.909 \mathrm{~mol} \mathrm{dm}{ }^{-3}\left(\left[\mathrm{G}_{R}^{-}\right]=\left[\mathrm{G}_{S^{-}}^{-}\right]=0.454 \mathrm{~mol} \mathrm{dm}{ }^{-3}\right.$ ). $[\mathrm{H}]=0.0909 \mathrm{~mol} \mathrm{dm}^{-3}$. $\left[\mathrm{G}^{-}\right][\mathrm{H}]=0.909: 0.0909 \mathrm{~mol} \mathrm{dm}^{-3}=10 .{ }^{\circ}$ amino acid methyl ester hydrochloride salt. 
Acknowledgments. This work was supported by a grantin-aid for the National Core Research Center Program from MOST and KOSEF (No. R15-2006-022-01001-0), and the Ministry of Information \& Communications. Korea. under the Information Technology Research Center (ITRC) Support Program.

\section{References and Notes}

1. Cram, D. J. Angew: Chem., Lht. Ed. Engl. 1988, 27. 1009.

2. Izatt. R. M.: Wann. T.: Hathaway. J. K.: Zhang. X. X.: Curtis. J. C.: Bradshaw. J. S.: Zhu. C. Y. J. Incl Phenom. 1994. 17. 157.

3. Grove. J. T.: Viski. P. J. Org Chem 1990. 55. 3628.

4. (a) Gasparrini. F: Misiti. D.: Borchardt A: Burger. M. T.: Still, W. C.J. Org. Chem 1995.60.4314. (b) Hvun, M. H. Bull. Korean Chem. Soc. 2005. 26, 1153. (c) Zholl. L.: Lin, Z: Reaner. R: Mao. B.: Ge. Z. Eletrophoresis 2007. 28. 2658.

5. (a) Chao. Y.: Cram. D. J. J. An. Chem. Soc. 1976. 98. 1015. (b) Breslow. R.: Czarnik. A. W.: Lauer. M.: Leppkes. R.: Winkler. J.: Zimmerman, S. J. Am. Chem. Soc, 1986, 108, 1969. (c) Talma. A. G.: Jouin, P: De Vries, J. G.; Troostwijk, C. B.; Werumeus Buning. G. H.: Waninge, I. K.: Visscher. I.: Kellogg. R. M. J. Am. Chem.
Soc. 1985. 107.3981

6. Zang. X. X.: Bradshaw. J. S.: Zzatt. R. M. Chent. Rev 1997.97.3313.

7. Sawada. M.: Takai. Y: Mizooku. H. Y.: Kakeuchi. K.: Amada. S. Kaneda. S. T.: Mizooku. T.: Kakeuchi, K.: Ueno, K.: Hirose. K. Tobe, Y; Naemura, K. J. Am Chem. Soc. 1995. 117,7726.

8. (a) Sawada. M; Takai. Y.: Yamada, H; Nishida, J; Kaneda. T:; Arakawa. R.: Okamoto. M.: Hirose. K.: Tanaka. T.: Naemura. K J. Chem. Sox. Perkin Trants. 2 1998. 701. (b) Sawada. M. Mass Spectron. Rev 1997. 16.81.

9. (a) Kim. J.-K.: Lee. J. G; Lee. S.: Seo. J. J; Hong. J. K; Suh, H Bull. Korean Chem. Soc. 2002, 23, 543. (b) Kim. J.-K.; Song. S. H; Kim. J. H.: Kim, T. H.: Kim, H. S:; Suh. H. Bull. Kowan Chent. Soc. 2006. 27. 1577. (c) Kim. T.-K.: Kiml. J. H.: Song. S. H.: Jung. O-S.: Sul. H. J. hoch Phenont 2007. 58. 187. (d) Suh. H.: Kim. J.-K.: Tung. I. S.: Lee. S. E.: Kang. S. W.: Park. T. S. Bull. Korean Chem. Soc. $1998,19.411$. (e) Jo. S. J.: Jin. Y. E.: Kim. J. H.; Sul, H. S. Bull Korean Chem. Soc, 2007, $28,2015$.

10. Behr. J.-P.: Girodeau. J.-M.: Hayward, R. C.: Lehn, J.-M; Sauvage. T.-P. Heh: Chim. Acta 1980. 63. 2096.

11. Gemel. C.: Folting. K.: Caulton. K. G. Ihorg. Chem. 20M0. 39. 1593

12. Kalinowski, H. O; Seebach. D; Crass. G. Angew: Chem., Int. Ed. Engl. 1975, $1+762$

13. Stewart J. J. P. J. Comput. Chem. 1989, 10.209. 